\title{
Crisis Standards of Care Implementation at the State Level in the United States
}

\author{
Colton Margus, MD; ${ }^{1}$ (i) Ritu R. Sarin, MD, EMDM, FACEP; ${ }^{2,3}$ Michael Molloy, MB, MCh, EMDM, \\ FRCEM, FRCSEd; ${ }^{2,3,4}$ (1) Gregory R. Ciottone, MD, FACEP, FFSEM ${ }^{2,3}$
}

1. Department of Emergency Medicine, Mount Sinai St. Luke's-Roosevelt, Icahn School of Medicine, New York, New York USA

2. Department of Emergency Medicine, Harvard Medical School, Boston, Massachusetts USA

3. Fellowship in Disaster Medicine, Department of Emergency Medicine, Beth Israel Deaconess Medical Center, Boston, Massachusetts USA

4. University College Dublin, Dublin, Ireland

Correspondence:

Colton Margus, MD

266 Indian Avenue

Middletown, Rhode Island 02842 USA

E-mail: cmargus@bidmc.harvard.edu

Conflicts of interest/funding: none

Keywords: crisis standards of care; disaster medicine; emergency medicine; pandemic planning; public health preparedness

Abbreviations:
COVID-19: coronavirus disease 2019
CSC: Crisis Standards of Care
SOFA: sequential organ failure assessment

Received: April 24, 2020

Revised: June 9, 2020

Accepted: June 15, 2020

\section{doi:10.1017/S1049023X20001089}

(C) The Author(s), 2020. Published by Cambridge University Press on behalf of World Association for Disaster and Emergency Medicine. This is an Open Access article, distributed under the terms of the Creative Commons AttributionNonCommercial-NoDerivatives licence (http:// creativecommons.org/licenses/by-nc-nd/4.0/), which permits non-commercial re-use, distribution, and reproduction in any medium, provided the original work is unaltered and is properly cited. The written permission of Cambridge University Press must be obtained for commercial re-use or in order to create a derivative work.

\begin{abstract}
Introduction: In 2009, the Institute of Medicine published guidelines for implementation of Crisis Standards of Care (CSC) at the state level in the United States (US). Based in part on the then concern for $\mathrm{H} 1 \mathrm{~N} 1$ pandemic, there was a recognized need for additional planning at the state level to maintain health system preparedness and conventional care standards when available resources become scarce. Despite the availability of this framework, in the years since and despite repeated large-scale domestic events, implementation remains mixed.
\end{abstract}

Problem: Coronavirus disease 2019 (COVID-19) rejuvenates concern for how health systems can maintain quality care when faced with unrelenting burden. This study seeks to outline which states in the US have developed CSC and which areas of care have thus far been addressed.

Methods: An online search was conducted for all 50 states in 2015 and again in 2020. For states without CSC plans online, state officials were contacted by email and phone. Public protocols were reviewed to assess for operational implementation capabilities, specifically highlighting guidance on ventilator use, burn management, sequential organ failure assessment (SOFA) score, pediatric standards, and reliance on influenza planning.

Results: Thirty-six states in the US were actively developing (17) or had already developed (19) official CSC guidance. Fourteen states had no publicly acknowledged effort. Eleven of the 17 public plans had updated within five years, with a majority addressing ventilator usage (16/17), influenza planning (14/17), and pediatric care (15/17), but substantially fewer addressing care for burn patients $(9 / 17)$.

Conclusion: Many states lacked publicly available guidance on maintaining standards of care during disasters, and many states with specific care guidelines had not sufficiently addressed the full spectrum of hazard to which their health care systems remain vulnerable.

Margus C, Sarin RR, Molloy M, Ciottone GR. Crisis standards of care implementation at the state level in the United States. Prehosp Disaster Med. 2020;35(6):599-603.

\section{Introduction}

During disasters, limitations in available resources not only strain existing health systems but also force uncomfortable complexity into how medical care should be provided to the community as a whole. Domestic events within the United States (US) have demonstrated how quickly these systems can be overwhelmed and even destroyed during times of disaster. ${ }^{1}$ In just the last 15 years, the US Gulf Coast states have experienced the Deepwater Horizon oil spill (2010), ${ }^{2}$ the West Fertilizer Company explosion (2013), ${ }^{3}$ and hurricanes Katrina (2005) and Harvey (2017) with lasting impact on the medical system..$^{4-6}$ Wildfires occurring throughout the Western US are now recognized to have long-term health consequences and impact on hospital systems. ${ }^{7}$ With more than 1,000 tornadoes in the US each year, major cyclones like the 2011 Joplin, Missouri tornado can critically threaten health care systems in addition to devastating communities. ${ }^{8}$ As the US, along with the rest of the world, currently faces the spread of novel coronavirus disease 2019 (COVID-19) and individual states turn to their existing pandemic response frameworks for guidance, appropriate standardization of how emergency medical care should be provided under crisis circumstances becomes all the more critical. ${ }^{9}$

In 2009, the H1N1 influenza pandemic galvanized interest across the globe in how health professionals might operate under more austere conditions, in which a dramatic surge in patients might impede the standard approach to providing quality medical care..$^{10,11}$ Recognizing the need to enable "specific legal/regulatory powers and protections for health 
care providers in the necessary tasks of allocating and using scarce medical resources and implementing alternate care facility operations," 12 the Institute of Medicine (Washington, DC USA) developed recommendations to assist health officials in creating standards of care in times of pervasive or catastrophic disaster. Now renamed the National Academy of Medicine, the Institute of Medicine defined Crisis Standards of Care (CSC) as "a substantial change in usual health care operations and the level of care it is possible to deliver, which is made necessary by a pervasive (eg, pandemic influenza) or catastrophic (eg, earthquake, hurricane) disaster." ${ }^{12}$ In 2012, the organization released a subsequent report providing further guidance on integrated development with governments, emergency health services (EMS), health care organizations, and health care providers to ensure that CSC planning and response occurs with sufficient coordination of care for escalating need. ${ }^{13}$

Externally, it is not clear how many states in the US have begun to implement guidance for their health care providers, despite the continued occurrence of disasters that stretch resources and care options across the nation. Given the implications for how similar efforts may be pushed forward on the international stage, this study seeks: (1) to determine how many US states have developed or are in the process of developing CSC plans; (2) to determine the extent of progress in this area over the last five years, ${ }^{14}$ and (3) to define what areas of medical care are most commonly addressed within these guidelines.

\section{Methods}

From December 2014 through May 2015, an internet-based crosssectional search was conducted for publicly available documents regarding CSC protocols of individual state Departments of Public Health, Hospital Preparedness, and Emergency Preparedness. If plans were unable to be found, state officials were contacted by email and phone. Protocols made available on the Assistant Secretary of Preparedness and Response (ASPR; Washington, DC USA) Communities of Interest website, as well as the Center for Disease Control's Office of Public Health Preparedness and Response (Atlanta, Georgia USA) listings, were also reviewed.

From August 2019 through March 2020, the internet-based search was repeated for a five-year comparison, again looking at state-specific CSC protocols and plans online. Where such documents were not found, additional effort toward locating publicly available CSC plans involved contacting the relevant state's Department of Health, or if available, Division of Emergency Preparedness and Response (or equivalent) by email and phone. A voice message was left whenever possible. For those states without government response through these means, state hospital associations were additionally contacted.

This study was limited to publicly available state CSC plans with additional focus on official government websites used by each state's Department of Health. Only plans explicitly claiming by name to be CSC protocols for a particular state, whether hosted on the state government's website or provided by state officials, were considered. This research was determined by the Beth Israel Deaconess Medical Center Committee on Clinical Investigations (CCI; Boston, Massachusetts USA) as not constituting human subjects research, as defined by both the Office for Human Research Protections (OHRP; Rockville, Maryland USA) and the Food and Drug Administration (FDA; Silver Spring, Maryland USA) within the US Department of Health and Human Services (HHS; Washington, DC USA).
Data collected included which states currently have publicly available CSC plans, which states are currently developing protocols, and which states are not currently engaged in any significant development process. In addition, public CSC plans were further evaluated to determine whether they: (1) rely heavily on influenza planning; (2) address ventilator usage; (3) use the sequential organ failure assessment (SOFA) score as part of their guidance; and (4) address other unique criteria, such as burn management and pediatric care.

All 50 states were discretely categorized into states with public CSC plans, with private CSC plans, with CSC plans currently under development, or without any significant effort toward CSC development. One final category included those states for which no information on CSC planning could be ascertained. In addition, existing CSC plans were evaluated for inclusion of several predetermined concepts: influenza planning, burn care, pediatric care, ventilator usage, and reliance on the SOFA score. All references to these concepts in the text of each CSC plan were evaluated individually for their relevance by the authors, in order to meet an appropriate threshold for inclusion and for discrete categorization.

\section{Results}

As of May $2015,84 \%$ of all US states were developing (26/50) or had released (16/50) guidance on CSC (Figure 1a). Eight states were at that time not yet engaged in the public development of CSC. Of the 16 states with publicly available guidance for their states, 13 had explicitly "living documents" that would continue to be edited and updated as guidance recommendations change. Despite expressing a commitment to one day produce state-level CSC plans, a majority of US states (34/50) did not have finalized plans readily accessible online for their practitioners.

As of March 2020, 72\% of all US states were developing (17/50) or had released (19/50) guidance on CSC (Figure 1b). Seventeen states had publicly available CSC plans, while two states reported having privately available plans. Nine states reported no current work toward providing CSC guidance at the state level. Despite online search, as well as both phone and email inquiry, CSC efforts were not able to be characterized for five states.

Over the five-year period, only 17 states had no change to their CSC efforts. The rest saw either an escalation or de-escalation in planning (Figure 2). For example, eight states that had been actively pursuing CSC plans were able to finalize their guidelines. Meanwhile, seven states without previous efforts were able to initiate CSC development, with three going so far as to release official public guidance within the five-year timeframe.

That progress, however, may be overstated. Seven states which had endorsed plans in 2015 had by 2020 returned to planning or ended efforts entirely, and six additional states no longer claimed to be developing CSC plans at all. In stark contrast with these reversals, it is worth noting that $65 \%$ of the states with publicly available CSC guidelines in 2020 (11/17) had updated their plans within the preceding five-year period.

The diversity of state approaches to CSC guidance becomes even more pronounced when the content of the 17 public plans is considered. Fourteen of the available protocols draw upon the individual state's existing influenza preparations. Sixteen deal with usage of ventilators and critical care resources. Thirteen of these plans support using the SOFA score to assist in decision making for resource allocation. Fifteen plans specifically address pediatric populations, while nine specifically address burn patients (Figure 3). 
(a)

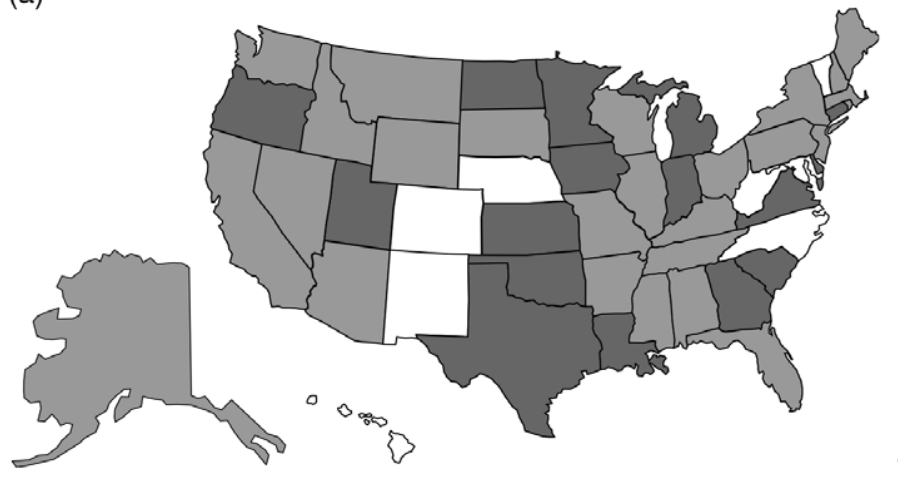

(b)

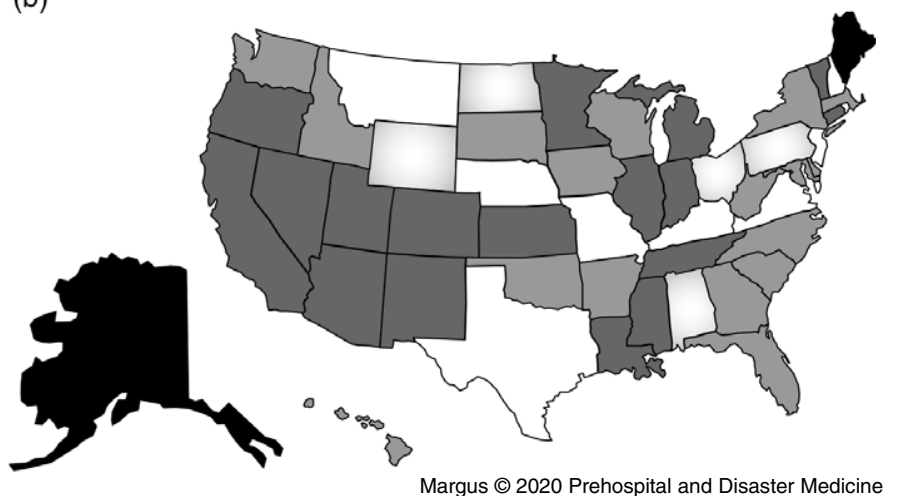

Figure 1. Confirmed US States Currently Developing (light grey) or Having Already Developed (public - dark grey; private black) CSC Guidance: (a) 2015 and (b) 2020.

Note: CSC efforts were not able to be characterized for five states (grey gradient).

Abbreviation: CSC, Crisis Standards of Care.

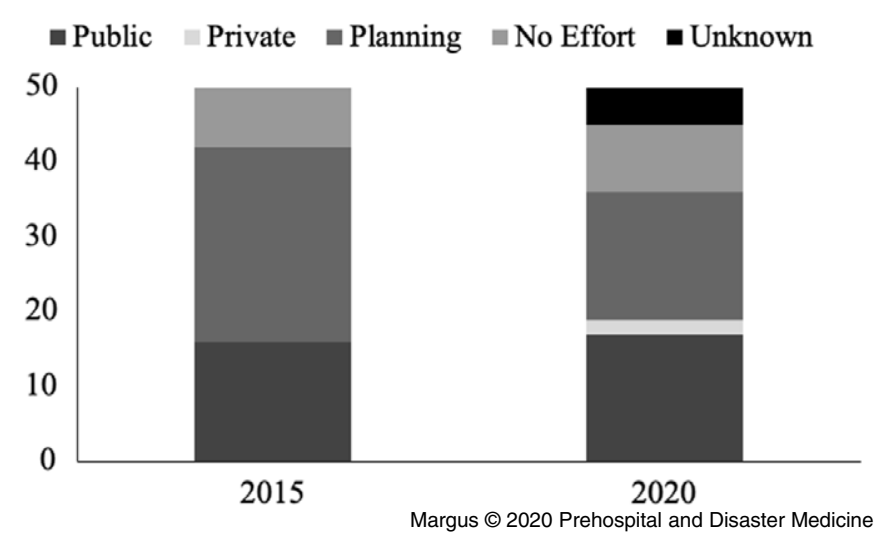

Figure 2. Implementation of State-Level CSC Guidance by Year $(n=50)$.

Note: States were designated as having public or private CSC plans, on-going CSC planning, no active effort toward CSC plans, or unknown CSC status.

Abbreviation: CSC, Crisis Standards of Care.

\section{Discussion}

Nine US states were not committed to developing CSC, while there were still five other states for which current efforts remained unknown. In 2020, this is a concerning number of states, given that the Institute of Medicine recommended early engagement and public adoption in 2009. ${ }^{12}$ Even more surprising, nine fewer states acknowledged CSC planning in 2020 than in 2015. Only one additional state had finalized CSC guidelines over the last five years, and it remained uncertain when, if at all, the remainder of states with plans in development would make their own documents publicly available.

A majority of public guidelines appeared to be structured on prior planning for an influenza-type outbreak following the 2006 Pandemic and All-Hazards Preparedness Act. ${ }^{15}$ Although the on-going COVID-19 pandemic garners significant attention, this focus on special pathogen risk may limit applicability should a future public health emergency primarily result in traumatic injuries, such as those occurring during mass-casualty bombings or earthquakes. Influenza-based guidance may not be as effective for clinicians seeking to triage surgical resources when systems are overwhelmed in this way.

And yet, even for those state guidance documents which substantively address influenza pandemic, two plans did not address ventilator usage or potential for shortages. With 30 influenza pandemics in the last 400 years suggesting significant hazard probability and severity, the University of Pittsburgh's Center for Biosecurity (Pittsburgh, Pennsylvania USA) estimated US demand for ventilators to reach $198 \%$ of capacity in the event of an outbreak comparable to the Spanish Flu of $1918 .{ }^{16}$ Addressing ventilator capacity would therefore seem to be of critical importance to maintaining standards of care during severe pandemic, let alone a crisis more broadly defined. ${ }^{17}$ Particularly in the on-going COVID-19 crisis, knowledge of ventilator surge capacity planning would serve to reassure hospitals and the public that there are resources in place should the standard infrastructure become overwhelmed.

The majority of CSC plans (12/17) relied heavily on the usage of SOFA scores to assist in decision making for resource allocation, as recommended in prior literature. ${ }^{18}$ However, it is important to note that the SOFA score was not developed as a tool to allocate scarce resources at the intersection of what is clinically and ethically appropriate, ${ }^{19}$ but instead to describe organ dysfunction and failure in sepsis. ${ }^{20}$ While the SOFA score's prevalence as a critical care tool to predict mortality may contribute to its use during times of crisis, other triage methods, such as the Simple Triage Scoring System (STSS) in the critical care literature, may have greater utility in directing resource allocation for influenza specifically. ${ }^{21}$ Furthermore, special populations, who are at greater risk of morbidity and mortality in times of disaster, will require more focused care strategies than what the broad application of triaging decision rules might otherwise allow. ${ }^{22}$

It is not possible based on the data collected in this research to explain the failure to implement state-level CSC guidelines across the country, or why the number of states in planning stages appears to have dropped over the last five years, despite continued urgency. Anecdotally, there are likely both competing public health priorities and perceived ethical dilemmas that make implementation difficult at the state level. For example, research efforts in public health emergency preparedness between 2009 and 2015 were largely focused on training utility, improved communications, sustainable response systems, analytical metrics, and addressing 


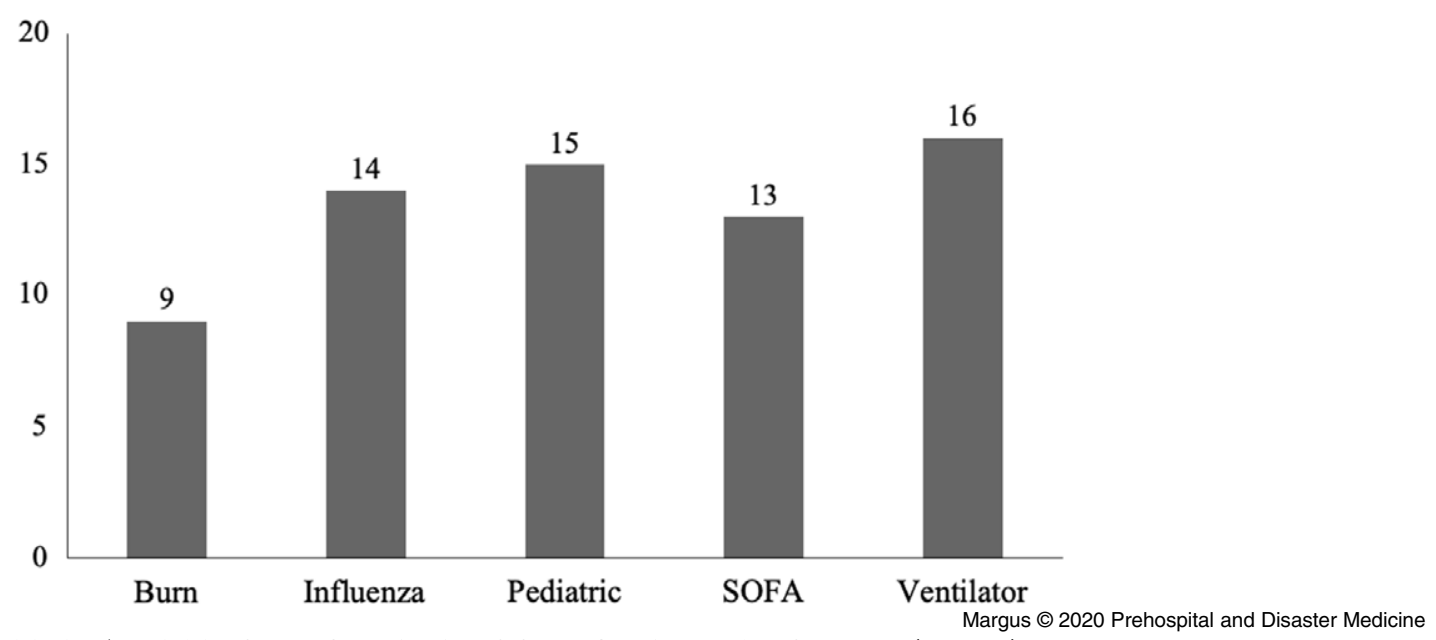

Figure 3. States with Publicly Available Crisis Standards of Care Guidance, by Content $(\mathrm{n}=17)$. Abbreviation: SOFA, sequential organ failure assessment.

vulnerable populations. ${ }^{23}$ These priorities demand attention but may not adequately address the immediate choices faced by clinicians in crisis scenarios, who find themselves suddenly asked by exceptional, catastrophic circumstances to act as both provider of individual patient care and allocator of scarce public health resources. $^{24,25}$

One state official conveyed that, while the state had initially been actively engaged in the CSC planning process, difficulties with "provider buy-in" and reliance on unfamiliar terminology were to blame for the effort's ultimate stagnancy. Interestingly, other state officials proffered distrust in a centralized, state-wide approach to crisis planning beyond what was already available, either at the national level or from the existing plans tailored for other states.

There remains substantial concern within the health care community that the very proposition of CSC inherently implies an aim to alter or even lower standards during a crisis rather than to maintain those standards in spite of that crisis. ${ }^{26}$ One perspective given is that crisis standards are unnecessary for, and may even be detrimental to, the making of sound allocation decisions in times of disaster, suggesting that such decisions do not place undo psychosocial or legal burden on individual providers. ${ }^{27}$ An alternative view however, is that the very nature of disasters in exceeding health care system capacity necessitates a framework that can maintain a standard of care despite unique liability concerns, resource limitations, and ethical complexities. ${ }^{28,29}$ Not in spite of that controversy but because of it, creating structured, regional strategies addressing potential obstacles to providing quality health care remains entirely within the scope and purpose of each state's preparedness endeavors.

The ethical principles guiding CSC development are not new, but, as the National Academy of Medicine recently suggested in its call for "developing tiered, proactive strategies" to face the threat of COVID-19, modern disasters and pandemics necessitate a cross-cutting, collaborative approach to addressing increasingly difficult health care management decisions. ${ }^{9,30}$ The World Health Organization (Geneva, Switzerland) has similarly recommended action "to reorganize and safely maintain access to high-quality, essential health services in the pandemic context" at both the national and local level. ${ }^{31}$

A decade has passed since the Institute of Medicine's first recommendation for CSC planning at state level, yet a significant number of states still lack specific care guidelines for their medical professionals in times of crisis. Furthermore, fewer states seem to be pursuing such guidelines currently than five years previously, suggesting that the plans have largely failed to achieve wider adoption within public health preparedness efforts. And while it is possible that an evolving pandemic threat may very well renew interest in state, national, and even international CSC strategies, the heavy reliance on existing influenza response frameworks may ultimately limit broader applicability to complex, large-scale disasters for which plans against a viral contagion do not readily translate.

Similar to the way in which the H1N1 pandemic galvanized the original Institute of Medicine recommendations for state-level coordination, the current global COVID-19 outbreak underscores the urgent need for systemic resilience when faced with insufficient medical resources to maintain conventional treatment standards. ${ }^{32}$ In this context, there is a clear space for greater commitment to developing CSC plans that can guide medical practitioners during times of disaster. The authors therefore recommend that government officials continue to engage in developing transparent CSC plans to promote greater public engagement ${ }^{33}$ as well as more candid dialogue on such difficult questions as resource allocation and ethical health care in times of crisis. The development of plans that can be utilized in scenarios such as a viral pandemic is recommended, but also beyond those of infectious etiology, and also that such plans be living documents that can be modified as medically significant recommendations for resource allocation are further developed and researched.

\section{Limitations}

A limitation of this study is that the search for state protocols was not done independently by multiple individuals, which could lead to inaccurate reporting. It is possible that the publicly available protocols reviewed by the authors are incomplete or are no longer the most current version being used, particularly in this current environment where plans may be changing rapidly.

A definitive answer as to whether a state has, or is in the process of developing, a CSC plan was not available for five US states. It may be argued that any plan not obtained through extensive search of online Department of Health resources, or through direct efforts to request such plans through email and phone contact, does not meet a threshold for what constitutes "public" availability. 
The extent to which states have pursued such plans and why some planning efforts came to fruition while others did not are not addressed in this study.

Additionally, the content of state CSC plans was evaluated in a binary way to determine simply whether a particular subject was addressed or not, making it difficult to draw broader conclusions. Furthermore, what is in the end written into a CSC plan regarding a subject may not indicate the full extent of its intellectual consideration in the plan's preparation.

Finally, this study limited its scope to a search only of publicly available protocols and to an analysis only of key plan components that could be operationalized under specific event scenarios. There may be more robust privately held living or developing protocols, at either the state or local level. Future research may address this. However, the goal of this study was to assess what guidance documents, if any, could become immediately available to address standards of care if such a crisis event were to occur in the respective state.

\section{Conclusion}

Despite existing national recommendations to the contrary for more than a decade, many US states do not yet provide public guidance on maintaining treatment standards in times of disaster. The challenges posed by COVID-19 may have rekindled interest in ethical health care resource stewardship, but the extent to which the on-going pandemic will enable greater government commitment to CSC planning for all hazards remains to be seen.

\section{Author Contributions}

$\mathrm{CM}$ and RS collected data and wrote the manuscript, while MM and GC provided additional expertise and vision. All authors reviewed the final manuscript.

\section{References}

1. Hick JL, Hanfling D, Cantrill SV. Allocating scarce resources in disasters: emergency department principles. Ann Emerg Med. 2012;59(3):177-187.

2. Ayer L, Engel C, Parker A, Seelam R, Ramchand R. Behavioral health of Gulf Coast residents 6 years after the Deepwater Horizon oil spill: the role of trauma history. Disaster Med Public Health Prep. 2019;13(3):497-503.

3. Metzger K, Akram H, Feldt B, et al. Epidemiologic investigation of injuries associated with the 2013 fertilizer plant explosion in West, Texas. Disaster Med Public Health Prep. 2016;10(4):583-590.

4. Schnall AH, Hanchey A, Nakata N, et al. Disaster-related shelter surveillance during the Hurricane Harvey response - Texas 2017. Disaster Med Public Health Prep. 2019;14(1):1-7.

5. Stephens W, Wilt GE, Lehnert EA, Molinari NM, LeBlanc TT. A spatial and temporal investigation of medical surge in Dallas-Fort Worth during Hurricane Harvey, Texas 2017. Disaster Med Public Health Prep. 2020;14(1):1-8.

6. Petrun Sayers EL, Parker AM, Ramchand R, Finucane ML, Parks V, Seelam R. Reaching vulnerable populations in the disaster-prone US Gulf Coast: communicating across the crisis lifecycle. J Emerg Manag. 2019;17(4):271-286.

7. Reardon S. Raging wildfires send scientists scrambling to study health effects. Nature. 2018;561(7722):157-158.

8. Houston JB, Spialek ML, Stevens J, First J, Mieseler VL, Pfefferbaum B. 2011 Joplin, Missouri tornado experience, mental health reactions, and service utilization: crosssectional assessments at approximately 6 months and 2.5 years post-event. PLoS Curr. 2015;7.

9. Hick JL, Hanfling D, Wynia MK, Pavia AT. Duty to Plan: Health Care, Crisis Standards of Care, and Novel Coronavirus SARS-CoV-2. Washington, DC USA National Academy of Medicine; 2020. https://nam.edu/duty-to-plan-health-carecrisis-standards-of-care-and-novel-coronavirus-sars-cov-2/. Accessed March 5, 2020.

10. Droogers M, Ciotti M, Kreidl P, et al. European pandemic influenza preparedness planning: a review of national plans, July 2016. Disaster Med Public Health Prep. 2019;13(3):582-592.

11. Kwan-Gett TS, Baer A, Duchin JS. Spring 2009 H1N1 influenza outbreak in King County, Washington. Disaster Med Public Health Prep. 2009;3(Suppl 2):S109-116.

12. Institute of Medicine (US) Committee on Guidance for Establishing Standards of Care for Use in Disaster Situations. Altevogt BM, Stroud C, Hanson SL, Hanfling D, Gostin LO, (eds). Guidance for Establishing Crisis Standards of Care for Use in Disaster Situations: A Letter Report. Washington, DC USA: National Academies Press; 2009.

13. Committee on Guidance for Establishing Crisis Standards of Care for Use in Disaster Situations; Institute of Medicine. Crisis Standards of Care: A Systems Framework for Catastrophic Disaster Response. Washington, DC USA: National Academies Press; 2012.

14. Sarin RR, Molloy MS, Ciottone GR. Analysis of state-level guidance on the implementation of crisis standards of care in the United States. Prehosp Disaster Med. 2015;30(S1):s154.

15. A bill to amend the Public Health Service Act with respect to public health security and all-hazards preparedness and response, and for other purposes: 'Pandemic and AllHazards Preparedness Act.' December 19, 2006. In: Public Law No: 109-417.
16. Bartlett JG, Borio L. Healthcare epidemiology: the current status of planning for pandemic influenza and implications for health care planning in the United States. Clin Infect Dis. 2008;46(6):919-925.

17. Powell T, Christ KC, Birkhead GS. Allocation of ventilators in a public health disaster. Disaster Med Public Health Prep. 2008;2(1):20-26.

18. Devereaux AV, Dichter JR, Christian MD, et al. Definitive care for the critically ill during a disaster: a framework for allocation of scarce resources in mass critical care: from a Task Force for Mass Critical Care summit meeting, January 26-27, 2007, Chicago, Illinois [USA]. Chest. 2008;133(5 Suppl):51S-66S.

19. Eastman N, Philips B, Rhodes A. Triaging for adult critical care in the event of overwhelming need. Intensive Care Med. 2010;36(6):1076-1082.

20. Vincent JL, Moreno R, Takala J, et al. The SOFA (sepsis-related organ failure assessment) score to describe organ dysfunction/failure. Intensive Care Med. 1996;22(7): 707-710.

21. Adeniji KA, Cusack R. The Simple Triage Scoring System (STSS) successfully predicts mortality and critical care resource utilization in $\mathrm{H} 1 \mathrm{~N} 1$ pandemic flu: a retrospective analysis. Critical Care. 2011;15:R39.

22. Dries D, Reed MJ, Kissoon N, et al. Special populations: care of the critically ill and injured during pandemics and disasters: CHEST consensus statement. Chest. 2014; 146(4Suppl):e75S-86S.

23. Savoia E, Lin L, Bernard D, Klein N, James LP, Guicciardi S. Public health system research in public health emergency preparedness in the United States (2009-2015): actionable knowledge base. Am J Public Health. 2017;107(S2):e1-e6.

24. Daniel M. Bedside resource stewardship in disasters: a provider's dilemma practicing in an ethical gap. J Clin Ethics. 2012;23(4):331-335.

25. Leider JP, DeBruin D, Reynolds N, Koch A, Seaberg J. Ethical guidance for disaster response, specifically around crisis standards of care: a systematic review. Am J Public Health. 2017;107(9):e1-e9.

26. Hodge JG, Hanfling D, Powell TP. Practical, ethical, and legal challenges underlying crisis standards of care. J Law Med Ethics. 2013;41(Suppl 1):50-55.

27. Schultz CH, Annas GJ. Altering the standard of care in disasters-unnecessary and dangerous. Ann Emerg Med. 2012;59(3):191-195.

28. Hanfling D, Hick JL, Cantrill SV. Understanding the role for crisis standards of care. Ann Emerg Med. 2012;60(5):669-670; author reply 670-671.

29. Kipnis K. Disasters, catastrophes, and worse: a scalar taxonomy. Camb Q Healthc Ethics. 2013;22(3):297-307.

30. Sauer LM, McCarthy ML, Knebel A, Brewster P. Major influences on hospital emergency management and disaster preparedness. Disaster Med Public Health Prep. 2009;3(2Suppl):S68-73.

31. WHO Global Team. Maintaining essential health services: operational guidance for the COVID-19 context. https://www.who.int/publications-detail/covid-19-operationalguidance-for-maintaining-essential-health-services-during-an-outbreak. Published March 25, 2020. Updated June 1, 2020. Accessed June 3, 2020.

32. Adalja AA, Toner E, Inglesby TV. Priorities for the US health community responding to COVID-19. JAMA. 2020. Epub ahead of print.

33. Timbie JW, Ringel JS, Fox S, et al. Allocation of scarce resources during mass casualty events. Evid Rep Technol Assess (Full Rep). 2012;(207):1-305. 